\title{
Compliance With Hepatitis B Vaccination Among Adult Males With Sexually Transmitted Infections
}

\author{
Jamshid Ayatollahi ${ }^{1}$; Ali Ayatollahi ${ }^{2}$; Reza Ayatollahi ${ }^{3}$; Ali Mellat Ardekani ${ }^{4}$; Seyed Hossein \\ Shahcheraghi ${ }^{1, *}$ \\ ${ }^{1}$ Infectious and Tropical Diseases Research Center, Shahid Sadoughi University of Medical Sciences, Yazd, IR Iran \\ ${ }^{2}$ Shahid Beheshti University of Medical Sciences, Tehran, IR Iran \\ ${ }^{3}$ International Campus, Shahid Sadoughi University of Medical Sciences, Yazd, IR Iran \\ ${ }^{4}$ Department of Neurology, Shahid Sadoughi University of Medical Sciences, Yazd, IR Iran \\ ${ }^{*}$ Corresponding author: Seyed Hossein Shahcheraghi, Infectious and Tropical Diseases Research Center, Shahid Sadoughi University of Medical Sciences, Yazd, IR Iran. Tel: +98- \\ 9132531389, E-mail: shahcheraghih@gmail.com
}

Received: March 6, 2013; Revised:June 6,2013; Accepted: April 9, 2014

\begin{abstract}
Background: Sexually transmitted infections(STIs) are a diverse group of infections caused by widely differing microorganisms (viruses, protozoa, bacteria, yeasts, ectoparasites and even a nematode), with transmission from person to person by sexual contact as its common characteristic. In all societies, sexually transmitted infections rank among the most common infectious diseases. These patients are at risk for hepatitis B virus infection, but have been relatively neglected in terms of hepatitis B virus vaccination.

Objectives: In this study, compliance with hepatitis B vaccination among adult males with sexually diseases was examined.

Patients and Methods: In this survey, 114 males, referred to an infectious diseases clinic, were asked to complete a questionnaire evaluating their knowledge of hepatitis B vaccine, and were interviewed to assess their reasons for refusal or acceptance. Finally, SPSS (version 16) was used to perform the statistical analyses. Variables were analyzed with Chi-square tests.

Results: The results of this study indicated that the overall hepatitis B vaccine acceptance rate was acceptably high. Among all, 53.5\% correctly identified that a vaccine to prevent hepatitis B virus infection had been available, $15 \%$ had a negative opinion, and $31.5 \%$ were neutral. The rations of all three doses of vaccination were $69.3 \%$.

Conclusions: Among the respondents, $87 \%$ were currently married, $86.8 \%$ were currently employed, $17.5 \%$ have addiction, $34.2 \%$ used condom, and $36.8 \%$ correctly identified hepatitis B as a sexually transmitted infection.
\end{abstract}

Keywords:Hepatitis B; Male; Sexually Transmitted Infection; Vaccination

\section{Background}

Sexually transmitted diseases (STDs), also referred to as sexually transmitted infections (STIs) and venereal diseases (VDs), have significant probabilities of transmission among humans through sexual behaviors. Diseases such as hepatitis B and HIV infections are most commonly transmitted by sexual activities. Many infectious diseases, including common cold, influenza, pneumonia, and most others that are transmitted person to person, can also be transmitted during sexual contact, if one person is infected, because of the involved close contact. However, even though these diseases may be transmitted during sex, they are not considered as STIs. Some STIs such as HIV infection can be transmitted from mother to child either during pregnancy or breastfeeding.

$\mathrm{HBV}$ (hepatitis B virus) is a noncytopathic virus and contains a partially double-stranded DNA genome. This virus predominantly infects hepatocytes and belongs to the hepadnavirus family. HBV has an outer envelope contain- ing hepatitis B surface antigen (HBsAg) and a core containing hepatitis B core antigen ( $\mathrm{HBcAg}$ ). Excess HBsAg is produced as subviral particles, which circulate in the blood and permit serological diagnosis of HBV. The core contains the genomic DNA as well as the virus-encoded DNA polymerase, detected in liver tissue. HBV also produces hepatitis B 'e' antigen or HBeAg, which is secreted into the blood and detected by serological assay (1-4). Hepatitis B is the only sexually transmitted disease with a safe and effective vaccine to protect against the infection. Many HBV carriers do not have symptoms and are not aware of the infection. When first infected; a person can develop an "acute" infection, which can range in severity from a very mild illness with few or no symptoms to a serious condition requiring hospitalization. Acute hepatitis B refers to the first six months after someone is exposed to HBV. Some people are able to fight the infection and clear the virus. For others, the infection remains

Implication for health policy/practice/research/medical education:

The implication of study is educating addicted people about hepatitis B virus vaccination. It is necessary to prevent progression and transfer of the disease to others.

Copyright @ 2014,Ahvaz Jundishapur University of Medical Sciences; Published by Kowsar Corp. This is an open-access article distributed under the terms of the Creative Commons Attribution License, which permits unrestricted use, distribution, and reproduction in any medium, provided the original work is properly cited. 
and leads to a chronic or lifelong illness. Until 1982, prevention of hepatitis B was based on passive immunoprophylaxis, but in 1982 the first vaccine for active immunization was prepared from purified HbsAg, derived from the plasma of a healthy HBsAg carrier (1-4). Transmission of $\mathrm{HBV}$ is resulted from exposure to infectious blood or body fluids containing blood. Possible forms of transmission include sexual contact, transfusion with human blood or blood products, reuse of contaminated needles and syringes, and vertical transmission from mother to child (MTCT) during childbirth.

Nowadays, Iran is classified as having low endemicity of hepatitis B infection. An investigation conducted in Iran revealed that $2.6 \%$ of hemodialysis patients suffered from HBV infection (1-4). One of the important priorities for elimination and control of HBV is to know the factors involved in HBV transmission, especially in endemic regions. Iran is the intermediate for $\mathrm{HBV}$ infection. The prevalence of $\mathrm{HBV}$ infection among blood donors have been reported in Iran. It is evident that 70\%-84\% of cirrhotic patients and $72 \%$ of individuals with hepatocellular carcinoma in Iran have evidence of exposure to HBV (1-4).

Improvement of the people's knowledge about HBV risk factors, national vaccination programs since 1993 for all neonates, and vaccination of high risk groups, might justify this decrease $(1,2)$. In 1987 , the former vaccine was supplanted by a genetically engineered vaccine derived from recombinant yeast (3). For pre-exposure prophylaxis against HBV in setting of frequent exposure (for example promiscuous heterosexuals), intramuscular (deltoid) injections of hepatitis $B$ vaccine are recommended; although today, recommendation is HBV vaccination of all adolescents to ensure coverage of individuals not immunized through infant immunization programs. HBV prevalence varies from intermediate to high in most countries of the Middle East, especially Iran $(4,5)$.

HBV can be transmitted among family members within households, possibly by contact of nonintact skin or mucous membrane with secretions or saliva containing HBV. However, at least $30 \%$ of the reported hepatitis $B$ infections among adults cannot be associated with identifiable risk factors. Although sexually transmitted disease transmission accounts for a relatively large proportion of incidental diseases in many countries, there continues to be little focus on males at risk for hepatitis B infection via sexual contact (6). Several vaccines have been developed for prevention of HBV infection. These rely on the use of one of the viral envelope proteins (HBsAg). The vaccine was originally prepared from plasma obtained from people with long-standing HBV infections. However, it is made using a synthetic recombinant DNA technology, which does not contain blood products. One cannot be infected with HBV through this vaccine. However, the studies on patients with sexually transmitted infections indicated relatively low rate of hepatitis B vaccine acceptance (7).

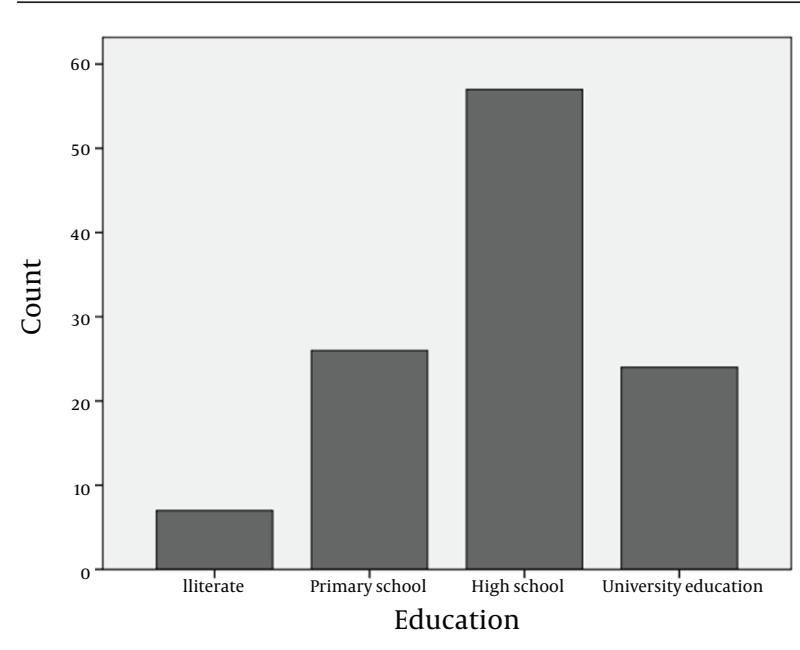

Figure 1. Educational Status

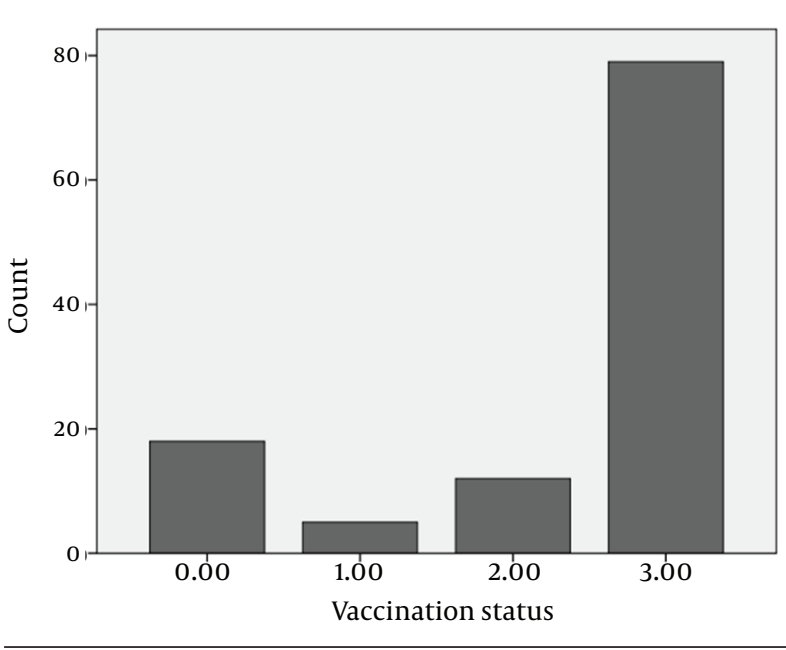

Figure 2. Vaccination Status

\section{Objectives}

In this study, compliance with hepatitis B vaccination among adult males with sexually transmitted diseases was examined.

\section{Patients and Methods}

This was a descriptive study. Survey questionnaires on the acceptance or reason of rejection of hepatitis B vaccine were distributed at an infectious diseases clinic. The reliability was tested by Cronbach's alpha method and validity was examined using three methods including factor analysis, concurrent validity, and content validity. The target population of this study was males (all of patients) with sexually transmitted infections, referred to our clinic in Yazd, IR Iran. A convenience sample of 114 adult males was recruited for this study. The responder completed a two-part questionnaire. The first part con- 
sisted of demographic data of the respondents (age, educational attainment, marital and addiction status), while the second part contained questions pertaining to the knowledge and practices of the patients on the use of hepatitis B vaccine.

We used the statistical package for social sciences (SPSS) version 16 to perform the statistical analyses. Variables were analyzed using chi-square tests. $\mathrm{P} \leq 0.05$ was accepted as statistically significant. Categorical variables ere age, education, employment, marital and addiction status, recognition of hepatitis B as a sexually transmitted infection, and status of hepatitis B vaccination.

\section{Results}

The 114 patients with sexually transmitted infection were 19-53 years old (mean: $31.92 \pm 8.58$ ), among which, $22.8 \%$ were not graduated from high school, $50 \%$ were graduated from high school, $21.1 \%$ had post-high school educations (Figure 1), and 6.1\% were illiterate; $87 \%$ were currently married, $86.8 \%$ were currently employed, $17.5 \%$ had addiction, and $34.2 \%$ used condom. Among the participants, $36.8 \%$ correctly identified hepatitis B as a sexually transmitted infection, and 53.5\% correctly identified that a vaccine to prevent hepatitis $B$ virus infection had been available. Vaccination statuses are shown in Figure 2 . The reasons for rejection of hepatitis B vaccination included: time constraints $(n=12)$, perceived low risk for infection ( $n=8)$, worries about privacy $(n=7)$, and concerns about side effects $(n=6)$.

\section{Discussion}

STI is a broader term than STD. An infection is colonization by a parasitic species, which may not cause any adverse effects. In a disease, the infection leads to impaired or abnormal function. In either case, the condition may not exhibit signs or symptoms. Increased understanding of infections, infecting most sexually active individuals, but causing disease in only a few, has led to increased use of the term STI (1). Public health officials originally introduced the term STI, which is increasingly used by clinicians alongside the term STD, to distinguish it from the former (1-4). As there has been no successful treatment for hepatitis B infection, the principal strategy for controlling hepatitis B infection is vaccination (8).

In Iran, recombinant hepatitis B vaccines are available at primary care centers free of charge to any applicant (9). This program entails vaccination of all neonates at birth, and routine immunization of high risk groups (e.g. health care worker). However, there continues to be little focus on adults at risk for hepatitis B infection through sexual transmission $(4,7,10,11)$. HBV is a hepadnavirus (hepa from hepatotropic (attracted to the liver) and DNA because it is a DNA virus) having a circular genome with partially double-stranded DNA. The HBV genome is unusual because the DNA is not fully double-stranded. The viruses replicate through an RNA intermediate formed by reverse transcription, which in practice relates them to retroviruses. Although replication takes place in the liver, the viruses spread to the blood, where viral proteins and antibodies against them are found in infected people. HBV is 50-100 times more infectious than HIV (5-7).

Acute infection with HBV is associated with acute viral hepatitis (an illness beginning with general symptoms); loss of appetite, nausea, vomiting, body aches, mild fever, and dark urine, and then progresses to development of jaundice. Itchy skin has been an indication as a possible symptom of all hepatitis virus types. The illness lasts for a few weeks and then gradually improves in most affected people. A few people may have more severe liver diseases (fulminant hepatic failure), and may die as a result. The infection may be entirely asymptomatic and remain unrecognized. Chronic infection with HBV may be either asymptomatic or associated with chronic inflammation of liver (chronic hepatitis), leading to cirrhosis over a period of several years $(5,6)$. This type of infection dramatically increases the incidence of hepatocellular carcinoma (liver cancer).

Chronic carriers are encouraged to avoid consuming alcohol as it increases their risk for cirrhosis and liver cancer. HBV has been linked to development of membranous glomerulonephritis (MGN). Symptoms outside the liver are present in 1\%-10\% of HBV-infected people and include serum-sickness-like syndrome, acute necrotizing vasculitis (polyarteritis nodosa), membranous glomerulonephritis, and papular acrodermatitis of childhood (Gianotti-Crosti syndrome). Serum-sickness-like syndrome occurs in the setting of acute hepatitis B, often preceding the onset of jaundice. Clinical features are fever, skin rash, and polyarteritis. The symptoms often subside shortly after the onset of jaundice, but can persist throughout the duration of acute hepatitis B. About $30 \%-50 \%$ of people with acute necrotizing vasculitis (polyarteritis nodosa) are HBV carriers.

HBV-associated nephropathy has been described in adults, but is more common in children. Membranous glomerulonephritis is the most common form. There are other immune-mediated hematological disorders, such as essential mixed cryoglobulinemia and aplastic anemia (1-5). HBV infection is a health problem worldwide, with approximately 400 million chronically infected people. Nowadays, Iran is classified as having low endemicity for hepatitis B infection. An investigation conducted in Iran revealed that $2.6 \%$ of hemodialysis patients suffered from HBV infection. The virus is transmitted by exposure to infectious blood or body fluids such as semen and vaginal fluids, while viral DNA has been detected in saliva, tears, and urine of the chronic carriers.

Perinatal infection is a major route of infection in endemic (mainly developing) countries. Other risk factors for developing HBV infection include working in a healthcare setting, transfusions, dialysis, acupuncture, tattooing, sharing razors or toothbrushes with an infected person, travel to endemic countries, and residence in 
an institution of endemic. However, HBVs cannot spread by holding hands, sharing eating utensils or drinking glasses, kissing, hugging, coughing, sneezing, or breastfeeding (4).

Hepatitis B vaccine was introduced via National Immunization Program (NIP) in Iran in 1993 (1). Sexual transmission is the most important mode of HBV spread in most developed countries and accounts for approximately $30 \%$ of acute HBV infections in the United States. Interestingly, in contrast to HIV, the risk of heterosexual transmission is greater when the infected individual is female than a male. Over $75 \%$ of the wives of male Iranian carriers have natural immunity against HBV. In 2004, an estimated 350 million individuals were infected worldwide. National and regional prevalence ranges from over $10 \%$ in Asia to under $0.5 \%$ in the United States and northern Europe. Routes of infection include vertical transmission (such as childbirth), early-life horizontal transmission (bites, lesions, and sanitary habits), and adult horizontal transmission (sexual contact, intravenous drug use). The primary method of transmission reflects the prevalence of chronic HBV infection in a given area. In low prevalence areas such as the continental United States and western Europe, injection drug abuse and unprotected sex are the primary methods of transmission, although other factors may also be important (1-4).

This study was the first survey in Iran to identify the acceptance and compliance of hepatitis B vaccination among the tested group. In our study, the acceptance rate of $69.3 \%$ was relatively and acceptably high for the infectious clinic patients. In studies in other countries, lower rates of acceptance, ranging from $23 \%$ to $69 \%$ (7, $10,11)$ were reported. One possible explanation for the higher rates in our survey could be the opportunity of free vaccination offered by the healthcare provider, not by the research worker. People may be more receptive when a healthcare worker recommends for immunization. We referred patients to a clinic which offered the vaccine to all the consecutive patients, as well as all neonates and children as routine healthcare manner, while in other studies, hepatitis B vaccine was only offered to the patients who agreed to participate to their survey, possibly making the process appear outside the routine vaccination scope. Those who refused the vaccine had significantly more addiction rates $(\mathrm{PV}=0.00)$, but considering other variables, those who accepted hepatitis B vaccination were not significantly different from those who refused it (for age group PV $=0.470$, for marital status $\mathrm{PV}=0.302$, for educational status $\mathrm{PV}=0.410$, for condom usage status $\mathrm{PV}=0.789$ ).

The reason given by patients for vaccination rejection often involved lack of knowledge about hepatitis B infection and importance of vaccination, fear of side effects, worries about privacy, perceived low risk for infection, and time situations. Survey of hepatitis B vaccine acceptance among other groups reported similar findings regarding health beliefs $(12,13)$. Some of these beliefs may be readily modified through brief group interventions, including providing accurate information about the risk for infection $(14,15)$. Acceptance of hepatitis B vaccination among healthcare staff were compatible with health thoughts $(16,17)$. Some of these beliefs were based on clarified information about the hazard of hepatitis B infection and the benefits of vaccination $(18,19)$. Studies that explain the indices corresponding to fellow vaccination (2nd, 3rd) by patients in infection referral clinics are few (20-22), so we suggest to establish a study to determine the health belief parameters about hepatitis $B$ vaccination acceptance in patients with STIs.

\section{Acknowledgements}

The authors gratefully acknowledge the personnel of Infectious and Tropical Diseases Research Center of Shahid Sadoughi University of Medical Sciences, Yazd, IR Iran.

\section{Authors' Contribution}

Jamshid Ayatollahi developed the original idea and the protocol; Ali Mellat and Ali Ayatollahi contributed to the development of the idea; Reza Ayatollahi and Seyed Hossein Shahcheraghi prepared the manuscript.

\section{Funding/Support}

The study was financially supported by Infectious and Tropical Diseases Research Center, Shahid Sadoughi University of Medical Sciences, Yazd, IR Iran.

\section{References}

1. Alavian SM, Gooya MM, Hajarizadeh B, Esteghamati A, Moeinzadeh A, Haghazali M, et al. Mass Vaccination Campaign against Hepatitis B in Adolescents in Iran: Estimating Coverage Using Administrative Data. Hepatitis Mon. 2009;9(3).

2. Jahangirnezhad M, Hajiani E, Makvandi M, Jalali F. A study on risk factors of chronic hepatitis B carriers. Jundishapur J Microbiol. 2011;4(4).

3. Wang CS, Chang TT, Yao WJ, Chou P. Comparison of hepatitis B virus and hepatitis $C$ virus prevalence and risk factors in a community-based study. Am J Trop Med Hyg. 2002;66(4):389-93.

4. Alavian SM. Hepatitis B virus infection in Iran; changing the epidemiology. Iran J Clin Infect Dis. 2010;5(1):51-61.

5. Poland GA, Jacobson RM. Clinical practice: prevention of hepatitis B with the hepatitis B vaccine. NEngl J Med.2004;351(27):2832-8.

6. Harris JL, Jones TS, Buffington J. Hepatitis B vaccination in six STD clinics in the United States committed to integrating viral hepatitis prevention services. Public Health Rep. 2007;122 Suppl 2:42-7.

7. Sansom S, Rudy E, Strine T, Douglas W. Hepatitis A and B vaccination in a sexually transmitted disease clinic for men who have sex with men. Sex Transm Dis. 2003;30(9):685-8.

8. Wilson BC, Moyer L, Schmid G, Mast E, Voigt R, Mahoney F, et al. Hepatitis B vaccination in sexually transmitted disease (STD) clinics: a survey of STD programs. Sex Transm Dis. 2001;28(3):148-52.

9. Zimet GD, Kee R, Winston Y, Perkins SM, Maharry K. Acceptance of hepatitis B vaccination among adult patients with sexually transmitted diseases. Sex Transm Dis. 2001;28(11):678-80.

10. Alavian SM, Zamiri N, Gooya MM, Tehrani A, Heydari ST, Lankarani $\mathrm{KB}$. Hepatitis $\mathrm{B}$ vaccination of adolescents: a report on the national program in Iran.J Public Health Policy. 2010;31(4):478-93.

11. Alavian SM, Hajarizadeh B, Ahmadzad-Asl M, Kabir A, BagheriLankarani K. Hepatitis B Virus Infection in Iran: A Systematic Re- 
view. Hepatitis Mon. 2008;8(4).

12. Weinstock HS, Bolan G, Moran JS, Peterman TA, Polish L, Reingold AL. Routine hepatitis B vaccination in a clinic for sexually transmitted diseases. Am J Public Health. 1995;85(6):846-9.

13. Sellors J, Zimic-Vincetic M, Howard M, Chernesky MA. Lack of compliance with hepatitis B vaccination among Canadian STD clinic patients: Candidates for an accelerated immunization schedule? CanJ Public Health. 1997;88(3):210-1.

14. Banatvala JE, Damme P. Hepatitis B vaccine - do we need boosters? J Viral Hepatitis. 2003;10(1):1-6.

15. Braka F, Nanyunja M, Makumbi I, Mbabazi W, Kasasa S, Lewis RF. Hepatitis B infection among health workers in Uganda: Evidence of the need for health worker protection. Vaccine. 2006;24(4748):6930-7.

16. Sukriti, Pati NT, Sethi A, Agrawal K, Agrawal K, Kumar GT, et al. Low levels of awareness, vaccine coverage, and the need for boosters among health care workers in tertiary care hospitals in India. $J$ Gastroen Hepatol. 2008;23(11):1710-5.

17. Nagao Y, Matsuoka H, Kawaguchi T, Ide T, Sata M. HBV and
HCV infection in Japanese dental care workers. Int J Mol Med. 2008;21(6):791-9.

18. Hoz F, Perez L, Wheeler JG, Neira M, Hall AJ. Vaccine coverage with hepatitis B and other vaccines in the Colombian Amazon: do health worker knowledge and perception influence coverage? Trop Med Int Health. 2005;10(4):322-9.

19. Zimet GD, Perkins SM, Winston Y, Kee R. Predictors of first and second dose acceptance of hepatitis B vaccine among STD clinic patients. IntJ STD \& AIDS. 2008;19(4):246-50.

20. Fatusi AO, Fatusi OA, Esimai AO, Onayade AA, Ojo OS. Acceptance of hepatitis B vaccine by workers in a Nigerian teaching hospital. East Afr Med J.2000;77(11):608-12.

21. Hafeez-ur-Rehman Mengal NH, Suwannapong N, Rajatanun T. Factors relating to acceptance of hepatitis B virus vaccination by nursing students in a tertiary hospital, Pakistan. J health, population, and nutrition. 2008;26(1):46.

22. Samoff E, Dunn A, VanDevanter N, Blank S, Weisfuse IB. Predictors of Acceptance of Hepatitis B Vaccination in an Urban Sexually Transmitted Diseases Clinic. Sex Transm Dis. 2004;31(7):415-20. 